\title{
Performance Analysis of Radio Access Technology Selection Algorithms in Heterogeneous 4G Networks
}

\author{
Marko Porjazoski $^{1}$, Ivana Todorovska ${ }^{1}$, Pero Latkoski ${ }^{1}$, Borislav Popovski ${ }^{1}$ \\ Faculty of Electrical Engineering and Information Technologies, Ss. Cyril and Methodeus University, Skopje, \\ Republic of Macedonia ${ }^{1}$
}

\begin{abstract}
In this paper is given a performance analysis of Radio Access Technology selection (RATS) algorithmsfor Long Term Evolution - Advanced (LTE-A) heterogeneous networks. The relevant performances are analysed using atwo dimensional Markov Model. Two RATSalgotithms are applyed:the first oneis simple, based only on the availability of the small cell connection, while the second one is based on two more criteria: user mobility and network load. The performance analysis shows that better results are obtained using the features provided in LTE-A, compared to the ones in previous technologies. The RATS algorithm influences the system performances as well. The results show that using the RATS algorithm based on two more criteria provides better perfotmances than the simpler one.
\end{abstract}

Keywords: Heterogeneous network, LTE-A, RATS algorithms, Markov Model, Video streaming

\section{INTRODUCTION}

Heterogonous wireless networks (HWN) are a solution to the increasing demand for mobile broadband bandwidth, required by the exponentially growing number of smartphones, tablets and other mobile devices which contribute to a huge expansion of wireless data traffic[1].

HWNs consist of classical cellular networks, composed of traditional high-power macro nodes, forming macro cells, that provide a full coverage of a wide area, and low-power nodes which offer overlay coverage in form of small cells, to provide extra capacity to certain areas within the microcells with a high traffic demand [2]. The purpose of HWNs is to offer users the best possible service, meaning that the user is served by the radio access technology (RAT) that satisfies their needs in the best way possible, taking care of the HWN surrounding at the same time.

Due to user's mobility, calls can be initiated in various cells or need to be handed over to another cell depending on the available coverage. It is important to pay attention to the possibility of radio link failure or handover failure [3], caused by different reasons, which significantly decreases the performances of the system.

In order to reduce these network failures, different algorithms for RATS, which are found in literature, can be implemented in such networks [4], depending on different criteria. Handover decision criteria including advantages and disadvantages of their use can be found in [5].

In this paper we will analyze the performances of a system that uses different configurations for 3GPP Release $10-$ LTE-A in both macro and small cells.
The study will include two different algorithms for RATS on new call arrival or user location change. The metrics that we are going to use in order to evaluate the system performances are: new call blocking probability, average number of active call users in the system and delay.

\section{SYSTEM MODEL}

In this paper we will study the performances of a HWN that consists of macro and small cells that use different configurations of LTE-A. First of all, we will set up the system model and we will define the parameters that we use for representing the performances of the system, for the two different RATS algorithms that will be used later.

It is important to be noted that if in a certain area there is only macro cell coverage, the user will be served by the macro cell, but if the user is located in the small cell coverage area, it is up to the RATS algorithm to decide whether the user will be served by the macro cell's or by the small cell's base station.

New call arrival rate is assumed to be a Poisson process with mean $\lambda_{\mathrm{n}}$ (calls/minute), while call duration is exponentially distributed with mean $1 / \mu$ (minutes), where $\mu$ is average call ending rate. Due to user mobility, handover occurs between two macro cells (horizontal handover - HHO), or between a small and a microcell in both directions (vertical handover- VHO). We consider that $\mathrm{HHO}$ and $\mathrm{VHO}$ are independent Poisson processes; mean HHO occurrence is denoted as $\lambda_{\mathrm{h}}$ and mean VHO occurrences from macro to small cell and from small to macro cell are denoted as $\lambda_{\mathrm{h}_{\_} \mu}$ and $\lambda_{\mathrm{h}_{\perp} \mathrm{M}}$, respectively. 


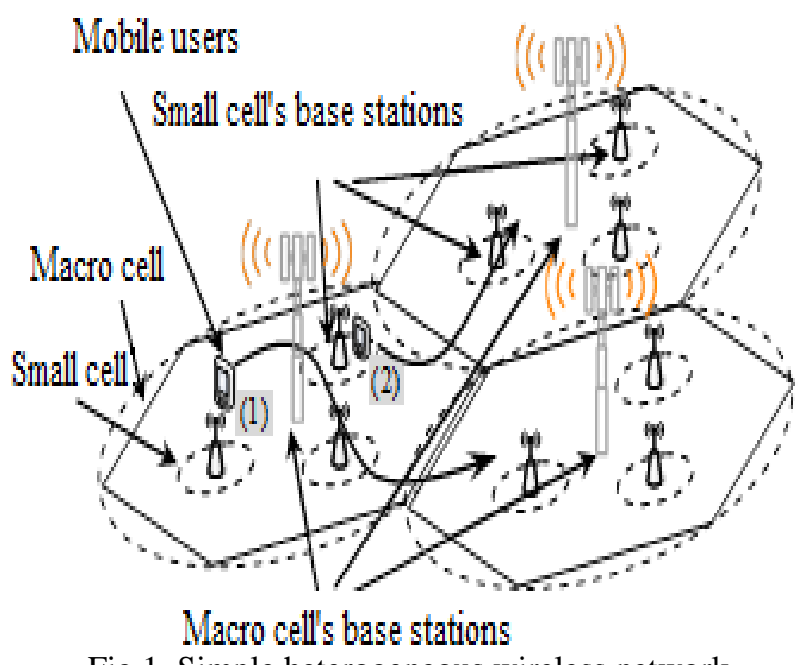

Fig 1. Simple heterogeneous wireless network

Handover occurrence depends on the time that the mobile user resides in the small or macro cell, i.e. small and macro cell dwell time.

\section{III.ANALYTICAL MODEL}

In order to evaluate and compare the system performances for different configurations of LTE-A, we use a two dimensional Markov model. A state in the Markov model is defined as the number of users with ongoing calls in both macro and small cells.

The parameters that define the transitions between two neighbour Markov states depend on the RATS algorithm. The number of states in both dimensions is delimited by the RAT used. In this paper we will use different configurations of LTE-A for both macro and small cells, including different channel bandwidths, use of Carrier Aggregation (CA) and use of Multiple Input Multiple Output (MIMO).

LTE-A not only supports $20 \mathrm{MHz}$ maximum channel bandwidth, but it also allows CA of up to 5 Component Carriers (CC) which leads to $100 \mathrm{MHz}$ maximum channel bandwidth. However, this channel bandwidth is not commonly used in practice, due to limited bandwidth available that the operators can use. The use of MIMO in means of spatial multiplexing enables additional available resources that can be used for supporting additional users. In [6] we analyze the LTE-A voice and video streaming capacity.

Using the method from [6] we can calculate the maximum number of calls that can be supported by one LTA-A cell, which will be used in the Markov model. Because the number of supported vide calls is lower than the number of voice calls, in order to keep the simplicity, in our analyses we will use video streaming capacity. The number of supported video calls per cell for different LTEA configurations is represented in Table 1.

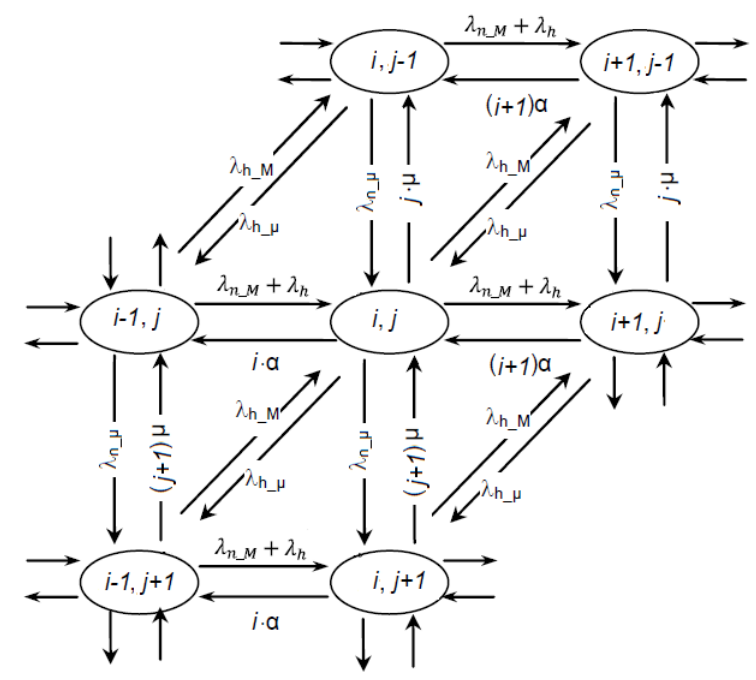

Fig 2. Two-dimensional Markov model

TABLE I NUMBER OF SUPPORTED VIDEO CALLS PER CELL

\begin{tabular}{|l|l|l|l|l|l|l|}
\hline \multirow{2}{*}{} & \multicolumn{2}{|c|}{ No CA } & \multicolumn{5}{|c|}{ CA for 20MHz: } \\
\cline { 3 - 7 } & \multicolumn{2}{|c|}{} & $\begin{array}{c}\text { 2C } \\
\text { C }\end{array}$ & $\begin{array}{c}\text { 3C } \\
\text { C }\end{array}$ & $\begin{array}{c}\text { 4C } \\
\text { C }\end{array}$ & 5CC \\
\hline $\begin{array}{l}\text { Channel } \\
\text { band- } \\
\text { width } \\
\text { [MHz] }\end{array}$ & 5 & 20 & 40 & 60 & 80 & 100 \\
\hline No MIMO & 34 & $\begin{array}{l}14 \\
5\end{array}$ & 291 & 437 & 584 & 729 \\
\hline 2x2 MIMO & 68 & $\begin{array}{l}28 \\
4\end{array}$ & 568 & 852 & $\begin{array}{l}113 \\
6\end{array}$ & 1420 \\
\hline 4x4 MIMO & $\begin{array}{l}13 \\
2\end{array}$ & $\begin{array}{l}54 \\
0\end{array}$ & $\begin{array}{l}108 \\
0\end{array}$ & $\begin{array}{l}162 \\
0\end{array}$ & $\begin{array}{l}216 \\
0\end{array}$ & 2700 \\
\hline
\end{tabular}

Figure 3 shows the Markov model we will use for analyzing the performances in the system we propose.

Each state in the Markov model is noted with an (i,j) pair, representing the number of channels occupied by macro and small cells, respectively. Using Table 1 we denote the maximum number of available channels for serving user calls in macro cells $-\mathrm{C} 1$ and small cells $-\mathrm{C}_{2}$.

There are three conditions that can cause necessity for acceptance of an additional call by a macro cell base station, which is represented by transition from a state in one column in the Markov model to a state in the neighbor right column. Two of these conditions are represented by horizontal transitions to right $\left(\lambda_{n-M}+\lambda_{h}\right)-a$ new call arrives in macro cell $\left(\lambda_{\mathrm{n} \_\mathrm{M}}\right)$ or a HHO occurs $\left(\lambda_{\mathrm{h}}\right)$, and the third one is represented by diagonal transition to right $\left(\lambda_{\mathrm{h}_{\_} \mathrm{M}}\right)$, which represents a VHO from a smallto macro cell. A necessity for an additional call acceptance by a small cell base station can occur when a new call in the small cell arrives $\left(\lambda_{n_{\Perp}}\right)$, which is represented by the vertical transition from a state in one row in the Markov model to the a state in the row below it, or when VHO occurs from a macro to a small cell $\left(\lambda_{\mathrm{h}_{\mu}}\right)$, which is represented by the diagonal transition in left from a state in the row above. 
Vol. 5, Issue 10, October 2016

A vertical transition to the state in the row above is caused by an ending call served by a small cell, which occurs with meani $\mu$, where $\mu$ is the average call ending rate and $i \in[0$, $\mathrm{C}_{2}$ ]. It should be noted that HHO between two small cells is not possible. A horizontal transition to the left can be caused due to two reasons: ending call served by a macro cell, which occurs with mean $\mathrm{j} \mu$ or a location change, which causes VHO from a macro to a small cell, denoted with $\mathrm{j} v$, where $v$ is macro cell boundary crossing rate and $\mathrm{j}$ $\epsilon\left[0, \mathrm{C}_{1}\right]$. This leads to representing the macro cell channel release rate as $\alpha=\mu+v$, so this transition is denoted as j $\alpha$.

The probabilities of the system being in each state can be calculated using the Markov model, which is represented in [7]. The values for solving the equilibrium equations depend on the RATS algorithm used and the previously proposed valuesfor all the parameters. In [7] and [8] three RATS algorithms are shown. We will shortly represent two of thesealgorithms in Section 4 and then in Section 5 we will analyze the results we acquired for our scenarios using the equations from [7] and [8].

\section{IV.RAT SELECTION ALGORITHMS}

A. "Always Small Cell” RATS Algorithm

The first RATS algorithm is a very simple one -"Always Small Cell". Figure 3 shows two block diagrams for this algorithm.

Fig 3. shows the case when a new call arrives to the area covered by a HWN. If there is small cell coverage, the call will be served by the small cell's base station, if not, it will be served by the macro cell's base station. Very similar scenario occurs when a user with an active call changes the location (Fig. 4) - if the new location is covered by a small cell, the call will be handed over to the small cell's base station, if not, it will be connected to the macro cell's base station.

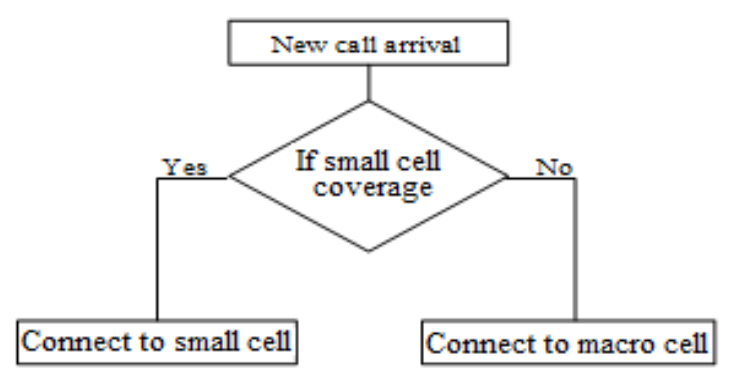

Fig 3. "Always Small Cell” Initial RATS algorithm

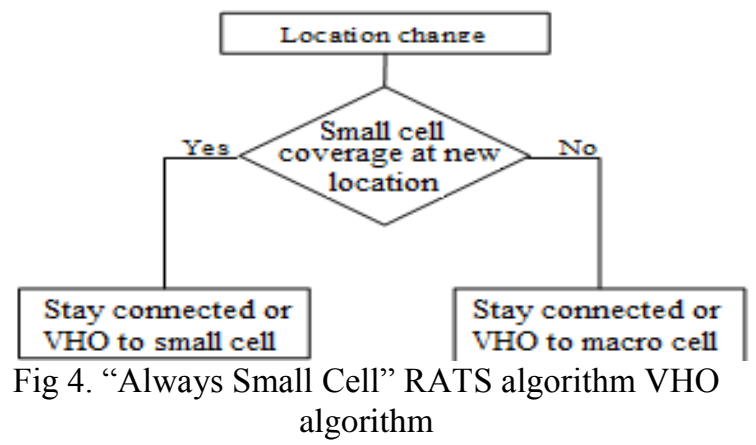

B. RATS algorithm based on user mobility and network load - "Mobility and Load"

The second RATS algorithm is more complicated and it is based on two more criteria: user mobility and network load ("Mobility and Load").

Similarly to the "Always Small Cell", when a new call arrives it is first checked if there is small cell coverage. If no small cell network is found, the user mobility type is checked. There are two mobility types: vehicular and nonvehicular. If the user is non-vehicular it is connected to the small cell. The vehicular users are subject to one more examination -the networks loads. If themacro cell's load is higher than the small cell's load, the service is established by the small cell and the other way around.

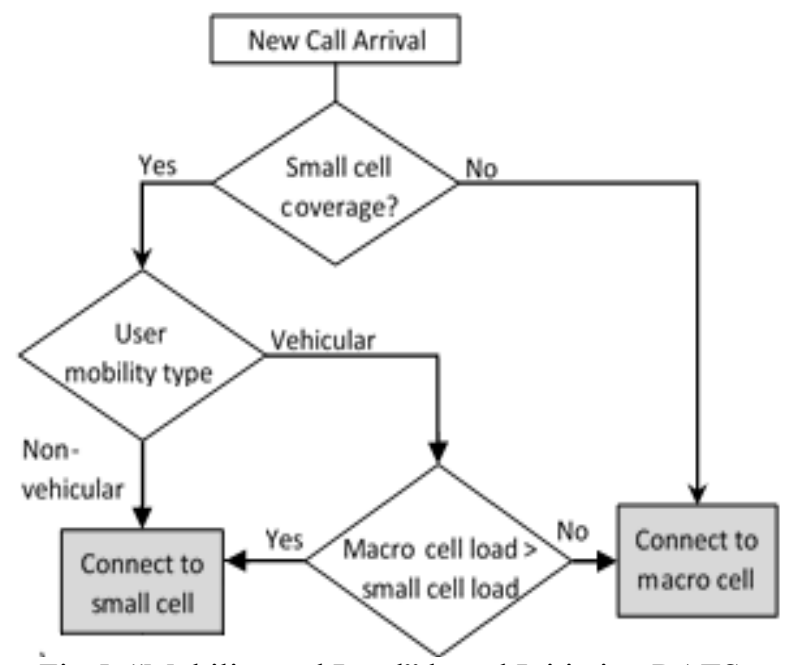

Fig 5. "Mobility and Load" based Initiation RATS algorithm

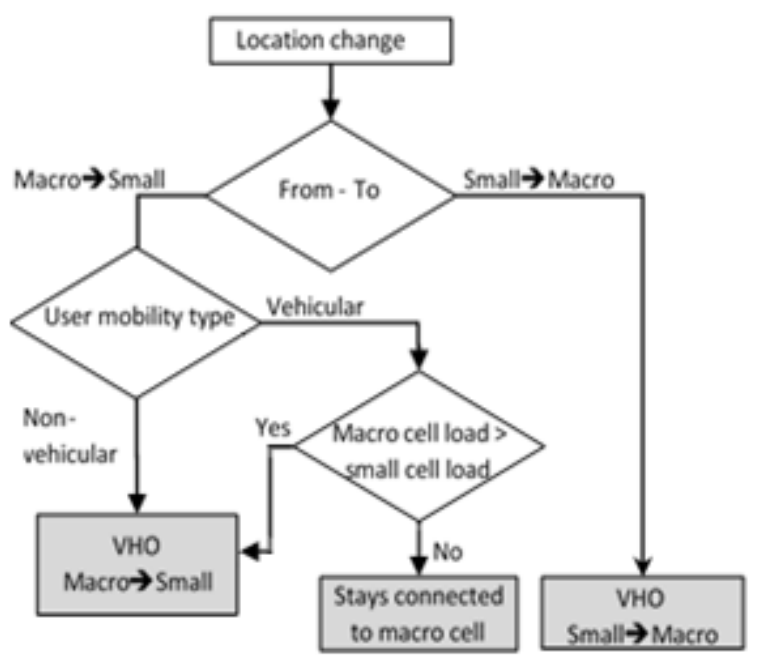

Fig 6. "Mobility and Load" VHO algorithm

The handover algorithm works similarly to the one previously described. When the user leaves the small cell zone of coverage, the call is automatically handed over to the macro cell. If the location change is the other way around - the user with an active call moves to an area where small cell connection is available, the mobility type 
Vol. 5, Issue 10, October 2016

is examined. Non-vehicular users are handed over to the small cell, whereas for vehicular users the networks loads are checked. If the macro cell's load is higher than the small cell's load the session is handed over the small cell. Otherwise it remains served by the macro cell.

\section{PERFORMANCE METRICS AND RESULTS}

The parameters that we have used in order to evaluate the system performances, for the both RATS algorithms are: new call blocking probability, average number of active call users in the system and delay [7].

The performances of the algorithms are examined under the following conditions: service type is a two way (uplink and downlink) $384 \mathrm{kbps}$ video, small to macro cell area ratio $c=0.1$, average call duration $1 / \mu=5 \mathrm{~min}$, average macro cell dwell time $1 / v=5 \mathrm{~min}$.

Maximum number of supported users in macro cell and small cell is given in Table 2. Furthermore, the call arrival rate, $\lambda_{n}$, is assumed to be in the range of $[5,100]$ calls per minute, while the percentage of vehicular users in the smallcell is $\theta=0.3$.

Table 2 shows scenarios that use different configurations of LTE-A features in the macro and small cells. Scenario 1 represents a case when the macro and the small cell both use $5 \mathrm{MHz}$ channel bandwidth, without the use of $\mathrm{CA}$ or MIMO, which can be compared to UMTS technology, which uses $5 \mathrm{MHz}$ channel bandwidth. As we go further, every scenario adds LTE-A features, including use of $20 \mathrm{MHz}$ channel bandwidth, CA and MIMO, which leads us to the scenario 5 - a network contained of $20 \mathrm{MHz}$ channel bandwidth and use of 2x2 MIMO technology in macro cells and $5 \mathrm{MHz}$ channel bandwidth using $2 \times 2$ MIMO in small cells.

TABLE 2 SCENARIOS THAT USE DIFFERENT CONFIGURATIONS OF LTE-A FEATURE IN THE MACRO AND SMALL CELLS

\begin{tabular}{|c|c|c|c|c|}
\hline & Macro cell & $\mathrm{C}_{1}$ & Small cell & $\mathbf{C}_{2}$ \\
\hline $\begin{array}{c}\text { Scenario } \\
1\end{array}$ & $\begin{array}{l}5 \mathrm{MHz}, \text { no } \\
\text { CA, no } \\
\text { MIMO }\end{array}$ & 34 & $\begin{array}{l}5 \mathrm{MHz}, \text { no } \\
\text { CA, no } \\
\text { MIMO }\end{array}$ & 34 \\
\hline $\begin{array}{c}\text { Scenario } \\
2\end{array}$ & $\begin{array}{l}5 \mathrm{MHz}, \text { no } \\
\mathrm{CA}, 2 \times 2 \\
\text { MIMO }\end{array}$ & 68 & $\begin{array}{l}5 \mathrm{MHz}, \text { no } \\
\text { CA, no } \\
\text { MIMO }\end{array}$ & 34 \\
\hline $\begin{array}{c}\text { Scenario } \\
3\end{array}$ & $\begin{array}{l}20 \mathrm{MHz}, \text { no } \\
\text { CA, no } \\
\text { MIMO }\end{array}$ & 145 & $\begin{array}{l}5 \mathrm{MHz}, \text { no } \\
\mathrm{CA}, \text { no } \\
\text { MIMO }\end{array}$ & 34 \\
\hline $\begin{array}{c}\text { Scenario } \\
4\end{array}$ & $\begin{array}{l}\text { 20MHz, no } \\
\text { CA no } \\
\text { MIMO }\end{array}$ & 145 & $\begin{array}{l}5 \mathrm{MHz}, \text { no } \\
\mathrm{CA}, 2 \times 2 \\
\mathrm{MIMO} \\
\end{array}$ & 68 \\
\hline $\begin{array}{c}\text { Scenario } \\
5\end{array}$ & $\begin{array}{l}\text { 20MHz, no } \\
\text { CA, 2x2 } \\
\text { MIMO }\end{array}$ & 284 & $\begin{array}{l}5 \mathrm{MHz}, \text { no } \\
\mathrm{CA}, 2 \times 2 \\
\text { MIMO }\end{array}$ & 68 \\
\hline
\end{tabular}

The following figures, Fig. 7 to Fig. 12, show the obtained results for comparing the five scenarios for both RATS algorithms, for all three parameters. Figure 5 represents the results for "Always Small Cell", while Figure 6 shows the results for "Mobility and Load" RATS algorithm.

As it is expected, all figures from Fig. 7 to Fig. 12 show that increasing the cells capacity using the features of LTE-A the performances are highly improved.

Comparing the five scenarios, from scenario 1 to scenario 5 in Fig. 7 and Fig. 10, we can conclude that new call blocking probability is decreased when using cells with higher capacity. In scenario 5, a low enough call blocking probability can be gained for a much higher call arrival rate then in scenario 1 . Using both RATS algorithms, for scenario 5, the call arrival rate can be increased up to 80 calls/minute and the new call blocking probability is still close enough to 0. Fig. 8 and Fig. 10 represent the average number of active call users in the system for both RATS algorithms. For a particular call arrival rate, different for all the scenarios, this number reaches its maximum value. In scenarios with greater cell capacity this curve has slower rise, allowing the system to support higher call arrival rate.

Finally, the delay decreases using scenarios with higher cell capacity. This is an expected system performance because when more resources are available, new calls can be accepted with lower delay.

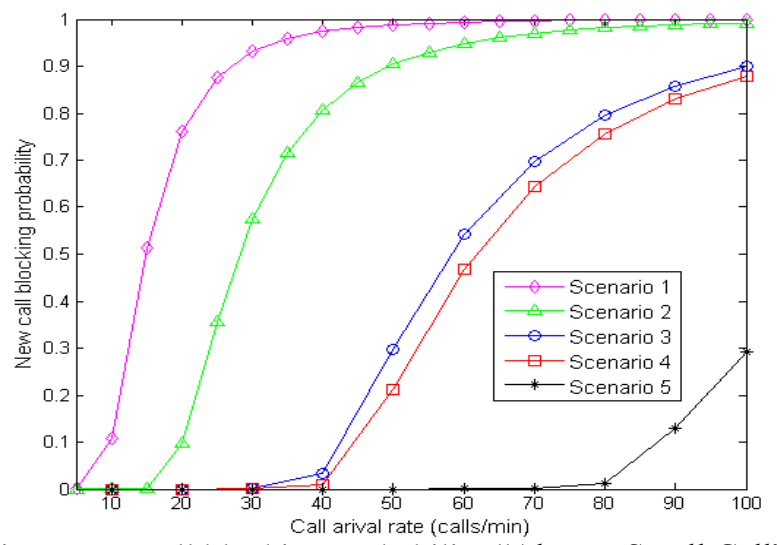

Fig 7. New call blocking probability "Always Small Cell" RATS algorithm

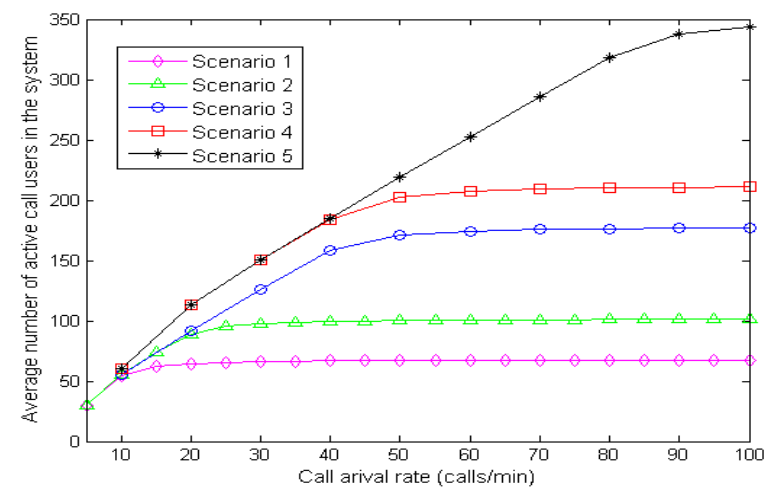

Fig. 8 Average number of active call users in the system for “Always Small Cell” RATS algorithm 


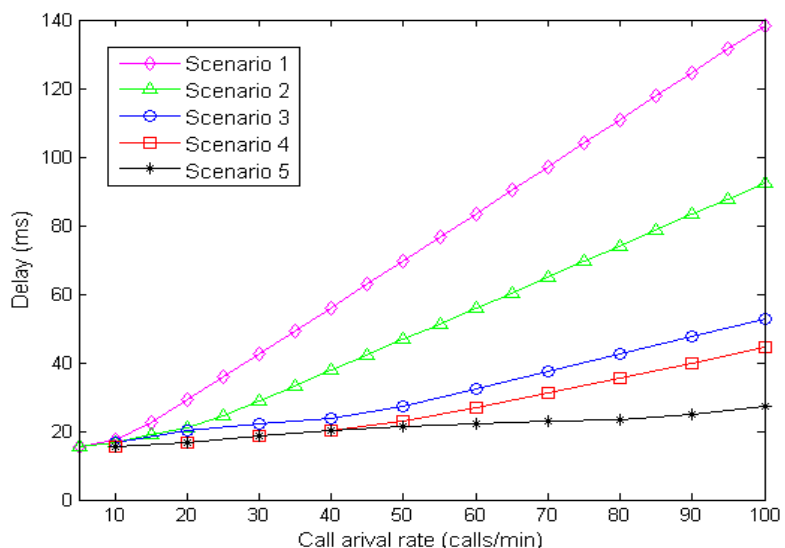

Fig. 9 Delay for "Always Small Cell” RATS algorithm

Additionally, having these results, we can compare the two RATS algorithms. Both RATS algorithms used decide which cell will be used for serving a call in a certain conditions. In "Mobility and Load" algorithm more conditions before making the decision are implemented in order to optimize the global system performances. This results into better performance results using the second algorithm that are represented on the figures.

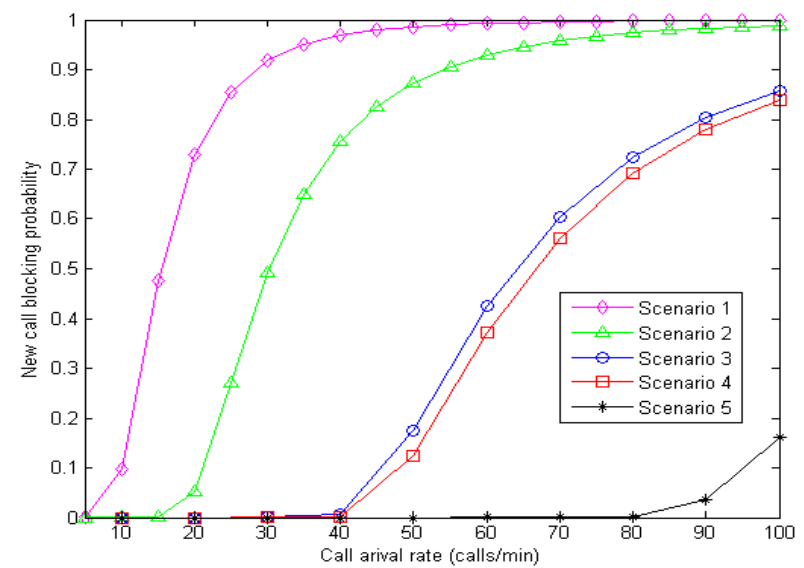

Fig. 9. New call blocking probability for "Mobility and Load" RATS algorithm

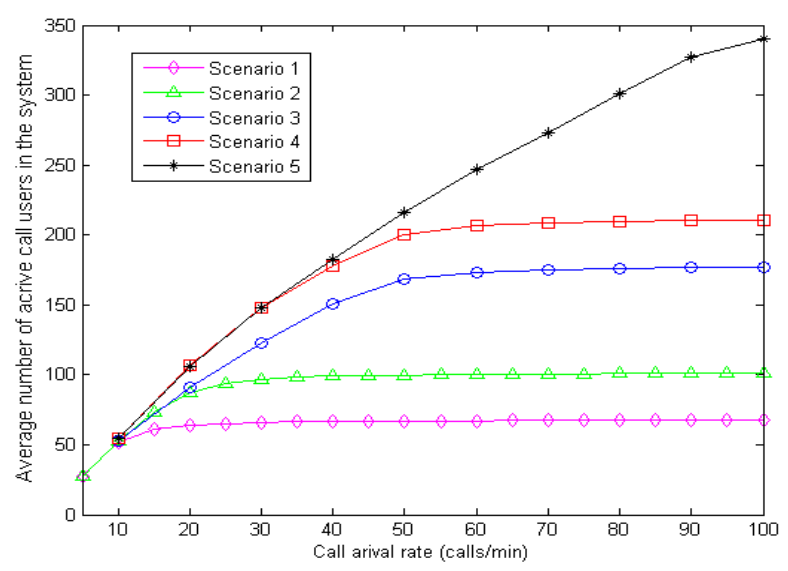

Fig. 10. Average number of active call users in the system for "Mobility and Load" based RATS algorithm

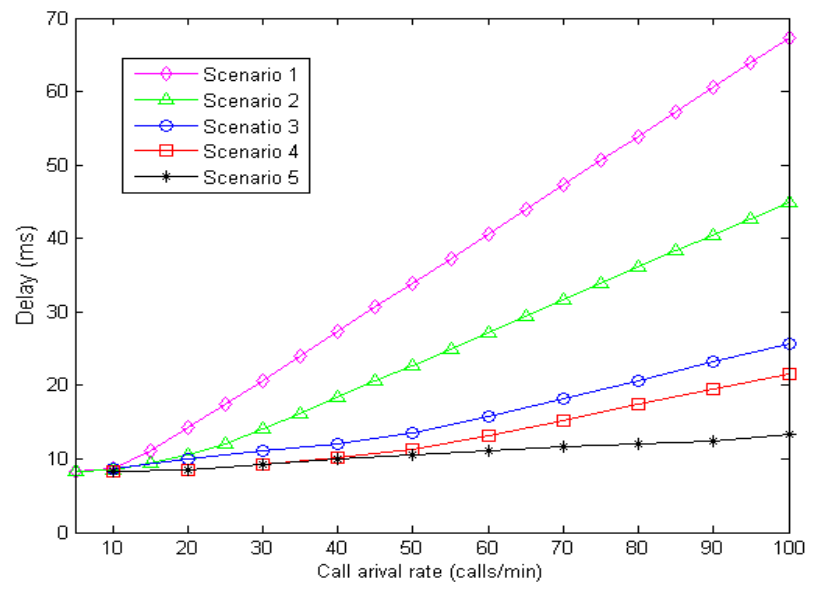

Fig. 11. Delay for "Mobility and Load" based RATS algorithm

Observing the results obtained for new call blocking probability, Fig 7 and Fig 10, the difference between the two RATS algorithms becomes noticeable for all the scenarios in conditions of lower call arrival rates. We can notice this in all the scenarios - when using "Mobility and Load" RATS algorithm, for the lower call arrival rates, the new call blocking probability is reduced, reaching the maximum decrease of almost two times.

Better results are gained due to the fact that more steps are used in the second decision making algorithm, which only allows call acceptance by the small cell if it is necessary. This means that if certain conditions are not satisfied, the call will be served by the macro cell.

The lower new call blocking probability is achieved as a result of the fact that the macro cell always uses higher capacity compared to the small cell, so as the difference between the capacities of the macro and the small cell grows, the difference in the new call blocking probability becomes more noticeable.

Fig. 8 and Fig. 11 present the average number of active call users in the whole system. The results do not differ much in both algorithms, because when using the same scenarios the system capacity remains the same. For certain call arrival rate, all the resources in the system become occupied so the system reaches the maximum number of users that can be supported.

The only difference that can be noticed between the results for the two different RATS algorithms is that using the second RATS algorithm this maximum is reached more slowly, due to the more optimized way of organizing the users between the cells.

Comparing Fig.9 and Fig. 12, a performances improvement can be noticed when using the "Mobility and Load" algorithm. The delay decreases due to the minor number of VHOs allowed by the second RATS algorithm, which is a result of the more complicated decision making. 


\section{VI.CONCLUSION}

Meeting the contemporary and upcoming data connection needs of the users that include rapid growth of the number of mobile devices and wireless data traffic, in this paper we introduced a system performance analysis for HWNs using the latest LTE-A technology.

In our work we compared three parameters that represent the system performances (new call blocking probability, average number of active call users in the system and delay) in five different scenarios while using the features of LTE-A RAT in both macro and small cells, applying two RATS algorithms.

Our results demonstrate the noticeable performance improvements using higher cell capacity which is enabled by the LTE-A features. Furthermore, the results manifest the major influence of using different RATS algorithms in such HWNs.

\section{REFERENCES}

[1] O. Stanze, A. Weber, "Heterogeneous Networks with LTEAdvanced Technologies", Bell Labs Technical Journal 18(1), 4158(2013), Wiley Periodicals, Inc.

[2] N. Lu, X. Zhu, Z. Jiang, X. Lu, F. Yang, Q. Bi, "Performance of LTE-Advanced Macro-Pico Heterogeneous Networks", 2013 IEEE Wireless Communications and Networking Conference (WCNC): MAC

[3] Y. Peng, W. Yang, Y. Zhang, Y. Zhu, "Mobility Performance Enhancements for LTE-Advanced Heterogeneous Networks", 2012 IEEE23rd International Symposium on Personal, Indoor and Mobile Radio Communications - (PIMRC)

[4] M. Zekri, B. Jouaber, D. Zeghlache, "A review on mobility management and vertical handover solutions over heterogeneous wireless networks", Computer Communications 35 (2012) 20552068

[5] D. Xenakis, N. Passas, L. Merakos, C. Verikoukis, "Mobility Management for Femtocells in LTE-Advanced: Key Aspects and Survey of Handover Decision Algorithms", IEEE Communications Surveys \& Tutorials, Vol, 16, NO. 1, First Quarter 2014

[6] M. Porjazoski, I. Todorovska, P. Latkoski, B. Poposki, "Estimation of VoIP and Video streaming capacity of LTE-Advanced", ETAI 2016, Ohrid, Macedonia, September 2016

[7] M. Porjazoski, B. Popovski, "Performance Analysis of Radio Access Technology Selection Algorithms in Heterogeneous Wireless Networks using 2-Dimesional Markov Model", International Journal of Research and Reviews in Next Generation Networks Vol. 1, No. 1, Science Academy Publisher, March 2011

[8] M. Porjazoski, B. Popovski, "Radio Access Technology Selection Algorithm for Heterogeneous Wireless Networks Based on Service Type, User Mobility and Network Load", TELSIKS. 